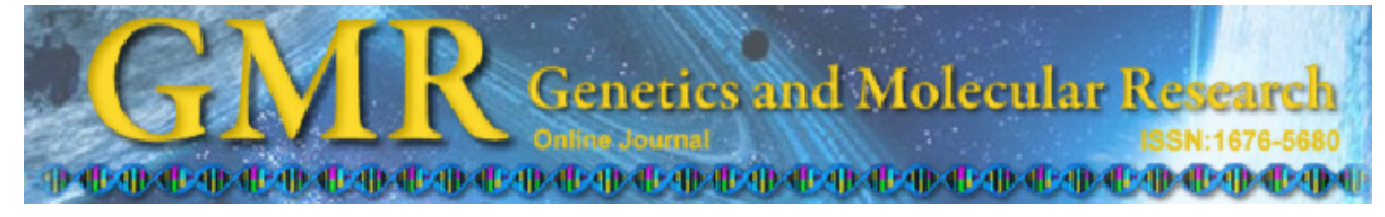

\title{
Identification and expression analysis of multiple FRO gene copies in Medicago truncatula
}

\author{
Ma. del C. Orozco-Mosqueda, G. Santoyo, R. Farías-Rodríguez, \\ L. Macías-Rodríguez and E. Valencia-Cantero \\ Instituto de Investigaciones Químico-Biológicas, \\ Universidad Michoacana de San Nicolás de Hidalgo, Morelia, \\ Michoacán, México \\ Corresponding author: E. Valencia-Cantero \\ E-mail: vcantero@umich.mx
}

Genet. Mol. Res. 11 (4): 4402-4410 (2012)

Received March 9, 2012

Accepted May 18, 2012

Published October 9, 2012

DOI http://dx.doi.org/10.4238/2012.October.9.7

\begin{abstract}
Iron $(\mathrm{Fe})$ is an essential element for plant growth. Commonly, this element is found in an oxidized form in soil, which is poorly available for plants. Therefore, plants have evolved ferric-chelate reductase enzymes (FRO) to reduce iron into a more soluble ferrous form. Fe scarcity in plants induce the FRO enzyme activity. Although the legume Medicago truncatula has been employed as a model for FRO activity studies, only one copy of the M. truncatula MtFRO1 gene has been characterized so far. In this study, we identified multiple gene copies of the MtFRO gene in the genome of M. truncatula by an in silico search, using BLAST analysis in the database of the M. truncatula Genome Sequencing Project and the National Center for Biotechnology Information, and also determined whether they are functional. We identified five genes apart from MtFRO1, which had been already characterized. All of the MtFRO genes exhibited high identity with homologous FRO genes from Lycopersicon esculentum, Citrus junos and Arabidopsis thaliana. The gene copies also presented characteristic conserved FAD and NADPH motifs, transmembrane regions and oxidoreductase signature motifs. We also detected expression in five of the putative $M t F R O$ sequences by semiquantitative RT-PCR analysis,
\end{abstract}


performed with mRNA from root and shoot tissues. Iron scarcity might be a condition for an elevated expression of the MtFRO genes observed in different M. truncatula tissues.

Key words: Medicago truncatula; FRO genes; Iron

\section{INTRODUCTION}

Iron $(\mathrm{Fe})$ is an essential element for plant growth; however, due to the low solubility of Fe in the soil, plants require effective mechanisms to obtain it. Based on the mechanism for Fe acquisition, plants can be grouped as either strategy I or strategy II plants (Römheld and Marschner, 1986). Strategy II plants include grasses whose roots secrete compounds known as phytosiderophores (PS) that chelate $\mathrm{Fe}^{3+}$ from the rhizosphere. Thereafter, the $\mathrm{Fe}^{3+}-\mathrm{PS}$ complex is introduced into the cell via the Yellow Stripe 1 (YS1) carrier protein in the plasmalemma (Römheld and Marschner, 1986). In contrast, strategy I plants are all higher plants (except grasses) and the acquisition of Fe occurs in 3 main reactions: i) proton excretion via a self-phosphorylated-type adenosine triphosphate (ATP)ase, thus acidifying the surrounding soil to increase Fe solubility; ii) reduction of $\mathrm{Fe}^{3+}$ to $\mathrm{Fe}^{2+}$ by a ferric chelate reductase (FRO) (Robinson et al., 1999; Schmidt, 1999), and iii) transport of $\mathrm{Fe}^{2+}$ by the iron-regulated transporter (IRT) through the plasmalemma membrane (Römheld, 1987; Eide et al., 1996).

It is known that iron deficiency promotes the excretion of phenolic compounds, organic acids, and flavins, which also contribute to Fe reduction and solubility (Welkie and Miller, 1988; Susín et al., 1994). The FRO gene, is a key enzyme in Fe acquisition, and the first FRO2 gene was identified in the model plant Arabidopsis thaliana (Robinson et al., 1999). Eight other copies of the $A$. thaliana (At)FRO gene family have also been identified and characterized; these genes exhibit tissue-specific expression (Wu et al., 2005), although there is some discrepancy in the expression patterns of different AtFRO copies in various tissues (Mukherjee et al., 2006). Other FRO genes have been reported in plants like Pisum sativum (Waters et al., 2002), Solanum lycopersicum (Holden et al., 1991), Citrus junos (Li et al., 2002), Cucumis sativus (Waters et al., 2002), Arachis hypogaea (Ding et al., 2009), and Oryza sativa (Gross et al., 2003).

Interestingly, it has recently reported the presence and expression of FRO genes in grasses such as barley and sorghum (Mikami et al., 2011), which raises the question of whether some other species of agricultural interest also present functional FRO genes, thus opening the possibility of improving their capabilities for iron acquisition. To date, no FRO activity has been observed in roots; this activity has only been detected in shoots and specific cellular organelles, thus likely contributing to Fe homeostasis and photosynthetic capacity (Mikami et al., 2011).

Medicago truncatula is a model legume that acquires Fe via strategy I. This plant has a small diploid genome, short generation time, is self-fertilizable, highly transformable, and its complete genome was recently reported (Cook, 1999; Trieu et al., 2000; Young et al., 2011). Additionally, it has the ability to establish symbiotic interactions with nitrogen-fixing bacteria of the genus Shizorhizobium, in addition to colonization by arbuscular mycorrhizal fungi (Harrison and Dixon, 1993). Recently it has been demonstrated that dimethyl hexadecylamine, an organic volatile compound produced by the rhizobacteria Arthrobacter agilis, induces the FRO activity in M. truncatula plants (Orozco-Mosqueda et al., 2012). So far only 1 copy of the M. truncatula (Mt)FRO1 gene has been characterized in this model plant (Andaluz et al., 
2009). To explore the possibility that more copies of $F R O$ genes are present and functional in the genome of M. truncatula, we carried out an in silico search for genes encoding FRO functions. Our results show that, apart from MtFRO1, there are at least 5 additional copies, which show highly conserved flavin adenine dinucleotide (FAD) and nicotinamide adenine dinucleotide phosphate-oxidase (NADPH) motifs, transmembrane regions, and oxidoreductase signature motifs, a feature of characterized FRO genes (Mukherjee et al., 2006; Andaluz et al., 2009). Additionally, MtFRO gene expression was confirmed by semiquantitative real-time polymerase chain reaction (RT-PCR) in conditions of Fe sufficiency and deficiency, showing a differential expression pattern. The identification of the MtFRO gene family opens the possibility to better understand the Fe uptake mechanisms in strategy I plants.

\section{MATERIAL AND METHODS}

\section{In silico search and identification of $F R O$ gene copies}

To search for unidentified FRO gene copies, we performed a homology Basic Local Alignment Search Tool (BLAST) search by employing the previously identified FRO1 gene sequence as a probe, obtained from GenBank with accession No. AY439088. The BLASTs were carried out in the database of the M. truncatula Genome Sequencing Resources at the following webpages: http://www.medicagohapmap.org, the latest version of the genome of $M$. truncatula (Mt3.5) and http://blast.ncbi.nlm.nih.gov. Other FRO sequences from P. sativum, $S$. lycopersicum, $C$. junos, $C$. sativus, A. hypogaea, and $O$. sativa were also employed in BLAST searches, although better results were obtained with the MtFRO1 sequence. We initially used Mt3.5 employing default parameters with an E-value of 0.01 , which was modified to become either more or less restricted in various searches to open the possibility of detecting homologous genes with low or high identity. Five FRO putative sequences (open reading frames) were obtained with different identities, which were depurated of introns with help of the GENSCAN program, available at http://genes.mit.edu/GENSCAN.html (The Board of Regents of the University of Wisconsin System, 2003). Prediction of transmembrane domains was performed using the Hidden Markov Model for TOpology Prediction (HMMTOP) 2.0 transmembrane topology prediction program at http://www.enzim.hu/hmmtop. The obtained putative gene sequences were also analyzed to search for FAD and NADPH domains, transmembrane regions, and the oxidoreductase signature motifs through BLAST; Conserved Domain Architecture (CDART; National Center for Biotechnology Information).

\section{Growth conditions of $M$. truncatula plants}

We used plants of M. truncatula ecotype Jemalong (A17-1). The seeds were scarified with concentrated anhydrous sulfuric acid. The excess of acid was removed by 6 washes with sterile deionized water and sterilization was carried out with a $12 \%$ sodium hypochlorite solution for $2 \mathrm{~min}$, rinsed with sterile distilled water, left for 3 to 4 days at $4^{\circ} \mathrm{C}$, then placed in complete Murashige-Skoog (MS) medium (with $0.6 \%$ fitagar) on Petri dishes. The Petri dishes were transferred to a Percival growth chamber (16-h light/8-h dark cycle), with a light intensity of $200 \mathrm{~mol} \cdot \mathrm{m}^{2} \cdot \mathrm{s}^{-1}$ at $22^{\circ} \mathrm{C}$ for 7 days. Subsequently, plants were transferred to Petri dishes containing MS with Fe sufficiency $(100 \mu \mathrm{M})$ and deficiency $(1 \mu \mathrm{M})$ and were kept in these conditions for $24 \mathrm{~h}$ in a growth chamber. 


\section{RNA extraction}

After $24 \mathrm{~h}$ of growth in Fe-sufficient or deficient media, total RNA extraction was carried out with the TRI reagent (Catalogue T9424, Sigma-Aldritch, St. Louis, MO, USA). The RNA was treated with RNase-free DNase I to remove residual genomic DNA (Mukherjee et al., 2006). The RNA samples were run on a $1.2 \%$ agarose gel, then stained with ethidium bromide to confirm the quality of the RNA. Finally, we carried out the cDNA synthesis with the SuperScript First-Strand Synthesis System for RT-PCR (Life Technologies/Gibco-BRL Grand Island, NY, USA).

\section{Semiquantitative RT-PCR conditions}

The oligonucleotides used in this study were designed with the Beacon Designer 4.02 program (Biosoft International Premier, Palo Alto, CA, USA). The PCR conditions were used as follows: initial denaturation at $95^{\circ} \mathrm{C}$ for $3 \mathrm{~min} ; 1 \mathrm{~min}$ at $95^{\circ} \mathrm{C}$ for denaturation, $1 \mathrm{~min}$ at $60^{\circ} \mathrm{C}$ for annealing, and $2 \mathrm{~min}$ at $72^{\circ} \mathrm{C}$ for extension for 30 cycles, and a final extension step at $72^{\circ} \mathrm{C}$ for $10 \mathrm{~min}$. PCR amplifications were performed with a TC-412 Techne Thermal Cycler (Keison Products Chelmsford, Essex, CM1 3UP, England). GoTaq ${ }^{\circledR}$ Master Mixes tubes (Promega Corporation Fitchburg, Madison, WI, USA) were used (tubes are supplied with enzyme, magnesium, dNTPs, and buffer). Only $0.1 \mu \mathrm{g}$ template cDNA and 50 $\mathrm{pM}$ of each primer were added to each tube.

\section{Sequencing of PCR products}

The RT-PCR products were additionally purified by using the Wizard ${ }^{\circledR}$ SV Gel and PCR Clean-Up System (Promega), following manufacturer instructions and were sequenced at the Laboratorio Nacional para la Diversidad Genomica, Centro de Investigación y de Estudios Avanzados-Irapuato, Mexico. The sequencing results were employed to corroborate the homology of the MtFRO genes by using the BLAST program as previously described.

\section{RESULTS}

\section{Identification of MtFRO gene copies}

In this study, we conducted an in silico search for multiple copies of $F R O$ genes in the genome of $M$. truncatula. To achieve this goal, we employed the previously identified MtFRO1 sequence as a probe in BLAST searches at the Genome Sequencing M. truncatula Resources database. The results revealed 5 additional sequences with high identity to $F R O$ genes (ranging from 67 to $76 \%$ ), and contained the highly conserved motifs for FAD (HPFT), NADPH (GPYG), transmembrane regions, and the oxidoreductase signature motif (LVMVCGGSGITPFIS), which are known characteristics of $F R O$ genes from diverse plants (Table 1). The number of transmembrane helices identified varied from 4 to 13 , depending on the gene that was analyzed. The $F R O 1$ gene encodes a protein of 703 amino acids (aa), while $F R O 2$ and $F R O 3$ sequences showed similar lengths of 792 and 740 aa, respectively. Notably, we found other sequences with shorter lengths, which we also named FRO4 
(476 aa), FRO5 (417 aa), and FRO6 (489 aa). FRO gene numbers were arbitrarily named according to the order in which they were found in our BLAST search. It is worth pointing out that we consider these smaller sequences as potential genes since they contain all of the motifs outlined above, as well as high identity with $F R O$ genes.

Table 1. Summarized features of the MtFRO family open reading frames (ORFs) and other ORFs from Arabidopsis thaliana and Pisum sativum used as a reference.

\begin{tabular}{lccllll}
\hline Gene & Size (aa) & Transmembrane regions & FAD motif & NADPH motif & Oxidoreductase motif & Reference \\
\hline MtFRO1 & 703 & 10 & WHPFTI & EGPYGP & LVMVSGGSGITPFIS & Andaluz et al., 2009 \\
MtFRO2 & 740 & 13 & WHPFSV & EGPYGH & LILVAGGIGLSPFLA & This study \\
MtFRO3 & 792 & 13 & WHPFTV & EGPYGP & IAMVSGGSGITPFIS & This study \\
MtFRO4 & 476 & 6 & WHPFTV & EGPYGP & IVMVSGGSGVTPFIS & This study \\
MtFRO5 & 417 & 4 & WHPFSV & EGPYGH & LILVAGGIGLSPFLA & This study \\
MtFRO6 & 489 & 6 & WHPFSI & DGPYGA & ILLVGLGIGATPLIS & This study \\
AtFRO1 & 704 & 10 & WHPFTI & EGPYGP & LVMVSGGSGITPFIS & Wu et al., 2005 \\
AtFRO2 & 725 & 10 & WHPFTI & EGPYGP & LVMVCGGSGITPFIS & Robinson et al., 1999 \\
PsFRO1 & 712 & 10 & WHPFTI & EGPYGP & LVMISGGSGITPFIS & Waters et al., 2002 \\
\hline
\end{tabular}

GenBank accession Nos. for the ferric chelate reductase (FRO) putative sequences: MtFRO1 (AY439088.1), MtFRO2 (XM_003594382.1), MtFRO3 (AC121237.19), MtFRO4 (AC121237.19), MtFRO5 (AC151000.3), MtFRO6 (AC154391.1), AtFRO1 (NM_100041), AtFRO2 (NM_100040.2), and PsFRO1 (AF405422.2). aa $=$ amino acid.

The above results suggest the possibility that the genome of $M$. truncatula contains multiple copies of the FRO gene, thus we decided to design primers to amplify them. The primer sequences that were used are found in Table 2. Once we amplified the putative $F R O$ sequences, the RT-PCR products were sequenced; of note, 5 of the 6 possible genes gave a positive result, except the gene sequence identified as FRO2. The sequencing results demonstrated that all of the amplified and partially sequenced genes showed $100 \%$ identity with the $M$. truncatula genome, as well as high identity with $F R O$ genes from other plant species, such as A. thaliana, L. esculentum, and C. junos (Table 3).

Table 2. List of primers designed and employed in this study.

\begin{tabular}{llc}
\hline Primer name & Nucleotide sequence $\left(5^{\prime}-\right.$-3' $\left.^{\prime}\right)$ & Size of the PCR expected product (bp) \\
\hline FRO1UPRT & TGTGTCGGTAGTTGTTGTTG & 207 \\
FRO1LORT & TCAGGTCTTGTTCCATAATGC & 165 \\
FRO2UPRT & GGTGGTATAGTGGTTGG & 145 \\
FRO2LORT & CATTTCTCTGACATTGATTC & \\
FRO3UPRT & AGGCGTTAGAGTGGAGCAAGAC & 210 \\
FRO3LORT & GAGAATGTAGAGATGGTGAGTGTAGAAG & 157 \\
FRO4UPRT & ATAACAAGCATTCCACAAATAAGG & 126 \\
FRO4LORT & AAGGCGAGCAGATAGTAACC & 126 \\
FRO5UPRT & TTATATTAGTGGCAGGTGGTATTGG & \\
FRO6UPRT & ACGGTTGAAAGAAGTGGAAGC & 209 \\
FRO6LORT & AAGACATAGAACAAGGAGTGGTAG & \\
MtACTINUPRT & TGATGAAGTGATTGAAGCATAGTG & \\
MtACTINLORT & CCAATAGGGACAACAACACTTTC & \\
\hline
\end{tabular}


Table 3. Sequence identity of the fragments of MtFRO genes amplified by RT-PCR compared with other identified $F R O$ genes ${ }^{\mathrm{a}}$.

\begin{tabular}{llcc}
\hline Gene name & Closest match & GenBank accession No. & Identity \\
\hline MtFRO1 & Medicago truncatula Fe(III)-chelate reductase (FROI) mRNA & AY439088.1 \\
MtFRO3 & Lycopersicon esculentum Fe(III)-chelate reductase (FROI) mRNA & AY224079.1 \\
MtFRO4 & Citrus junos Fe(III)-chelate reductase mRNA & DQ985810.1 & 99\% \\
MtFRO5 & Arabidopsis thaliana AtFRO6 Fe(III)-reductase mRNA & NM_124351.3 & $81 \%$ \\
MtFRO6 & Arabidopsis thaliana Fe(III)-reductase-like mRNA & NM_114450.2 & $71 \%$ \\
MtACT2 & Medicago truncatula ACT2 gene, promoter and exon 1 & AJ809891.1 & $74 \%$ \\
\hline
\end{tabular}

${ }^{a}$ All the sequences amplified by RT-PCR showed $\geq 99 \%$ identity with their own sequences found in silico (see Table 1).

\section{The multiple MtFRO copies are modulated by iron availability}

Once we identified the different copies of putative MtFRO genes and confirmed them by sequencing and BLAST identity, we explored whether these gene sequences are regulated. We performed semiquantitative RT-PCR to determine their expression in roots and shoots of plants grown under conditions of Fe sufficiency and deficiency. The results suggest that all of the sequences identified in this study, except $F R O 2$, are functional at the level of expression. After designing 3 different sets of oligonucleotides to detect $F R O 2$ expression, we were unable to detect a band by semiquantitative RT-PCR (data not shown). In addition, we also observed that expression of the different MtFRO copies is inducible in Fe-deficient growth conditions and might be tissue-specific (Figure 1). In particular, MtFRO1 was basally expressed in Fe-sufficient conditions, but when the plants are transferred to Fe-deficient media we noted an elevated expression level, especially in shoots. It was interesting to note that in conditions of Fe sufficiency, expression of the MtFRO3, MtFRO4, MtFRO5, and MtFRO6 genes was detected in shoots but not in roots. However, under Fe deficiency conditions the expression of these genes was increased in both root and shoot parts. This suggests that the genome of $M$. truncatula contains multiple functional $F R O$ gene copies, and that their expression pattern is likely tissue-specific and dependent on the concentration of $\mathrm{Fe}$ in the medium.

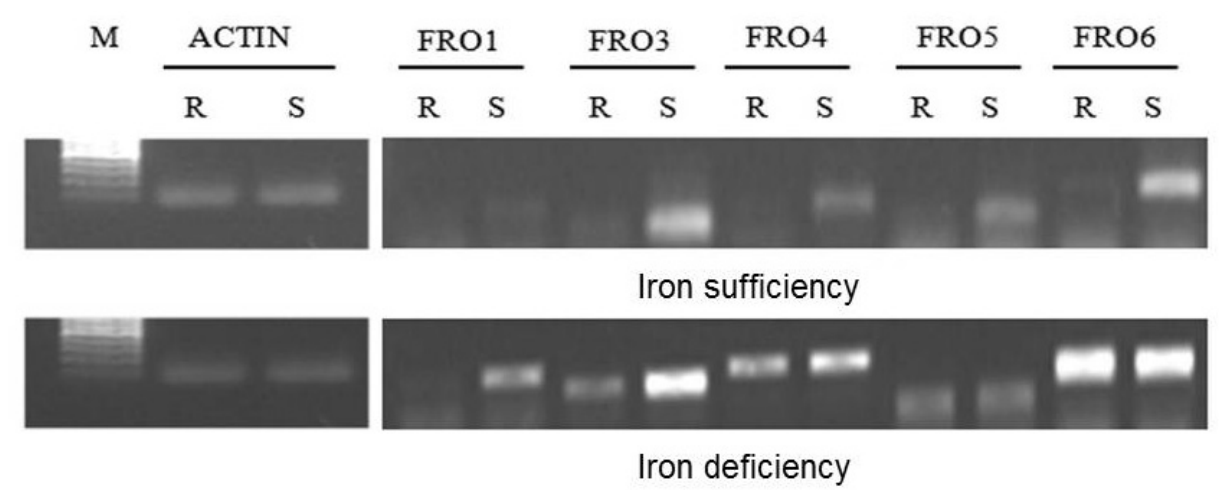

Figure 1. Semiquantitative RT-PCR detection of MtFRO genes in Medicago truncatula roots and shoots. M. truncatula plants were grown under iron sufficiency $(100 \mu \mathrm{m})$ and deficiency $(1 \mu \mathrm{m})$. The actin gene was employed as a control. Lane $M=$ molecular marker; $\mathrm{R}=$ roots: $\mathrm{S}=$ shoots. 


\section{DISCUSSION}

Plant genomics allows us to identify and further characterize genes that are involved in diverse cellular processes, such as Fe deficiency stress responses. Fe is one of the most abundant elements on earth, although its availability may be limited in plants; therefore, plants have developed efficient mechanisms for Fe acquisition (Römheld and Marschner, 1986). It is generally accepted that there are 2 strategies for Fe acquisition in plants. M. truncatula is a strategy I plant, which can acidify the rhizosphere and increase the bioavailability of $\mathrm{Fe}$ (Orozco-Mosqueda et al., 2012). In the present in silico study, we identified 5 putative sequences with high identity to FRO genes in the genome of M. trucatula (apart from FRO1, which was previously described; Andaluz et al., 2009). All sequences described herein showed highly conserved motifs characteristic of FRO proteins, such as FAD, NADPH, transmembrane regions, and the oxidoreductase signature motifs (Table 1). FRO genes are present in various plant species and are responsible for reducing $\mathrm{Fe}^{3}$ to $\mathrm{Fe}^{2}$, a more soluble form that can taken up by the roots (Hell and Stephan, 2003). Apart from FRO, other proteins such as IRT1, which is a high affinity transporter for $\mathrm{Fe}^{2+}$, play an essential role in Fe acquisition in plants such as $A$. thaliana (Varotto et al., 2002). The expression of FRO2 and IRTI is regulated by Fe deficiency conditions in strategy I plants (Connolly et al., 2003).

The FRO1 gene was the first to be characterized in A. thaliana (Robinson et al., 1999), 7 other functional copies of the same family have been characterized (Wu et al., 2005; Mukherjee et al., 2006). In this sense the question arises: why do plants such as $A$. thaliana or M. truncatula contain multiple $F R O$ copies in their genomes? One answer has been widely discussed in various studies, suggesting that the existence of multiple copies of the $F R O$ gene are required for $\mathrm{Fe}$ reduction, uptake, and homeostasis in different plant tissues or organs, which are differentially regulated by the availability of $\mathrm{Fe}$ (or other factors) (Wu et al., 2005; Mukherjee et al., 2006). Likewise, it has been postulated that the expression of $F R O$ genes in plant roots may play other roles, apart from reduction of Fe (Muherjee et al., 2006), since other elements can regulate their gene expression.

The presence of $F R O$ genes is not unique to strategy I plants, as other grasses such as rice contain 2 FRO genes (Ishimaru et al., 2007). It has been argued that $O$. sativa $(O s) F R O 2$ and OsFRO1 may not code for ferric chelate reductase functions, even when these activities have been reported only in mesophyll cells in other plant grasses such as barley and sorghum (Mikami et al., 2011). Interestingly, the expression of FRO1 and FRO2 genes in barley and sorghum was detected in leaves but not in roots. The above results suggest the possibility of detecting more gene copies in other grasses, and that the FRO genes may encode for FRO proteins with functions not only in leaves or shoots but in roots, which would break the exclusivity of Fe acquisition strategy I for non-grass plants.

In this study, we report evidence that the multiple copies of $F R O$ genes in M. truncatula are expressed in low Fe concentrations and are present in root and/or shoots. This agrees with other studies in A. thaliana, which demonstrated that genes such as AtFRO2 and AtFRO3 are expressed under low Fe conditions mainly in roots, while AtFRO5, AtFRO6, AtFRO8, and AtFRO7 are exclusively found in shoots and not regulated by this condition (Wu et al., 2005). Besides, the expression of each gene is tissue specific; for example, AtFRO2 and AtFRO3 are mainly expressed in roots, while AtFRO5 and AtFRO6 were detected in shoots and flowers, AtFRO6 and AtFRO7 in cotyledons and trichomes, and AtFRO8 is specifically expressed in 
veins of leaves (Wu et al., 2005), although slight differential expression has been proposed by other authors (Mukherjee et al., 2006). In M. truncatula, we also observed a differential expression pattern for each MtFRO gene with respect to the Fe condition. In conditions of Fe sufficiency, MtFRO1, MtFRO4, and MtFRO5 showed expression that can be considered basal, but MtFRO3 and MtFRO6 showed a strong expression level at the shoots. In Fe-deficient conditions, all of the MtFRO genes showed increased expression at both the roots and the shoots. It was very interesting to note the strong expression level of the MtFRO3 and MtFRO6 in the shoots, since experiments suggest that the ferric-chelate reduction is performed in aerial tissues. The role of the chelate $\mathrm{Fe}^{3+}$-nicotianamine on the plant $\mathrm{Fe}$ transport via the phloem has been demonstrated (Takahashi et al., 2003), and it has been suggested that Fe could be internalized in the cells via the $\mathrm{Fe}^{3+}$-nicotianamine transporter in both strategy I and strategy II plants (Koike et al., 2004). Our results provided molecular evidence that the FRO is strongly expressed in aerial tissues and indicates that $\mathrm{Fe}$ is reduced before it can be internalized in the plant cells as the results of Wu et al. (2005) and Mukherjee et al. (2006) suggest. Our study also showed that different members of the MtFRO genes are expressed in the same organs (roots, leaves, and stems), thus their physiological functions could be redundant. Further studies regarding the role of FRO for plant iron homeostasis utilizing loss-of-function mutants must now consider these redundant genes. We are currently conducting experiments to determine the iron-reducing functions of the multiple $M t F R O$ copies in different tissues, as well as their regulation by different factors, including the presence of plant growth-promoting rhizobacteria.

\section{ACKNOWLEDGMENTS}

Research supported by Consejo Nacional de Ciencia y Tecnología, México (\#128341) and Coordinación de la Investigación Científica-Universidad Michoacana de San Nicolás de Hidalgo (\#2.22). Ma. del C. Orozco-Mosqueda received a Doctoral Scholarship from ConacytMéxico.

\section{REFERENCES}

Andaluz S, Rodriguez-Celma J, Abadia A, Abadia J, et al. (2009). Time course induction of several key enzymes in Medicago truncatula roots in response to Fe deficiency. Plant Physiol. Biochem. 47: 1082-1088.

Connolly EL, Campbell NH, Grotz N, Prichard CL, et al. (2003). Overexpression of the FRO2 ferric chelate reductase confers tolerance to growth on low iron and uncovers posttranscriptional control. Plant Physiol. 133: 1102-1110.

Cook D (1999). Medicago truncatula: A model in the making! Curr. Opin. Plant Biol. 2: 301-304.

Ding H, Duan L, Wu H, Yang R, et al. (2009). Regulation of AhFRO1, an Fe(III)-chelate reductase of peanut, during iron deficiency stress and intercropping with maize. Physiol. Plant 136: 274-283.

Eide D, Broderius M, Fett J and Guerinot ML (1996). A novel iron-regulated metal transporter from plants identified by functional expression in yeast. Proc. Natl. Acad. Sci. U. S. A. 93: 5624-5628.

Gross J, Stein RJ, Fett-Neto AG and Fett JP (2003). Iron homeostasis related genes in rice. Genet. Mol. Biol. 26: 477-497. Harrison MJ and Dixon RA (1993). Isoflavonoid accumulation and expression of defense gene transcripts during the establishment of vesicular arbuscular mycorrhizal associations in roots of Medicago truncatula. Mol. Plant Microbe Interact. 6: 643-654.

Hell R and Stephan UW (2003). Iron uptake, trafficking and homeostasis in plants. Planta 216: 541-551.

Holden MJ, Luster DG, Chaney RL, Buckhout TJ, et al. (1991). Fe-chelate reductase activity of plasma membranes isolated from tomato (Lycopersicon esculentum Mill.) roots: comparison of enzymes from Fe-deficient and Fesufficient roots. Plant Physiol. 97: 537-544.

Ishimaru Y, Kim S, Tsukamoto T, Oki H, et al. (2007). Mutational reconstructed ferric chelate reductase confers enhanced 
tolerance in rice to iron deficiency in calcareous soil. Proc. Natl. Acad. Sci. U. S. A. 104: 7373-7378.

Koike S, Inoue H, Mizuno D, Takahashi M, et al. (2004). OsYSL2 is a rice metal-nicotianamine transporter that is regulated by iron and expressed in the phloem. Plant J. 39: 415-424.

Li L, Fan Y-H, Luo X-Y, Pei Y, et al. (2002). Expression of ferric chelate reductase gene in Citrus junus and Poncirus trifoliata tissues. Acta Bot. Sin. 44: 771-774.

Mikami Y, Saito A, Miwa E and Higuchi K (2011). Allocation of Fe and ferric chelate reductase activities in mesophyll cells of barley and sorghum under Fe-deficient conditions. Plant Physiol. Biochem. 49: 513-519.

Mukherjee I, Campbell NH, Ash JS and Connolly EL (2006). Expression profiling of the Arabidopsis ferric chelate reductase (FRO) gene family reveals differential regulation by iron and copper. Planta 223: 1178-1190.

Orozco-Mosqueda Ma del C, Velázquez-Becerra C, Macías-Rodríguez ML and Santoyo G (2012). Arthrobacter agilis UMCV2 induces iron acquisition in Medicago truncatula (strategy I plant) in vitro via dimethylhexadecylamine emission. Plant Soil DOI: 10. 1007/s11104-012-1263-y.

Robinson NJ, Procter CM, Connolly EL and Guerinot ML (1999). A ferric-chelate reductase for iron uptake from soils. Nature 397: 694-697.

Römheld V (1987). Different strategies for iron acquisition in higher plants. Physiol. Plant. 70: 231-234.

Römheld V and Marschner H (1986). Evidence for a specific uptake system for iron phytosiderophores in roots of grasses. Plant Physiol. 80: 175-180.

Schmidt W (1999). Mechanisms and regulation of reduction-based iron uptake in plants. New Phytol. 141: 1-26.

Susín S, Abían J, Peleato ML and Sanchez-Baeza F (1994). Flavin excretion from roots of iron-deficient sugar-beet (Beta vulagris L.). Planta 193: 514-519.

Takahashi M, Terada Y and Nakai I (2003). Role of nicotianamine in the intracellular delivery of metals and plant reproductive development. Plant Cell. 15: 1263-1280.

Trieu AT, Burleigh SH, Kardailsky IV, Maldonado-Mendoza IE, et al. (2000). Transformation of Medicago truncatula via infiltration of seedlings or flowering plants with Agrobacterium. Plant J. 22: 531-541.

Varotto C, Maiwald D, Pesaresi P, Jahns P, et al. (2002). The metal ion transporter IRT1 is necessary for iron homeostasis and efficient photosynthesis in Arabidopsis thaliana. Plant J 31: 589-599.

Waters BM, Blevins DG and Eide DJ (2002). Characterization of FRO1, a pea ferric-chelate reductase involved in root iron acquisition. Plant Physiol. 129: 85-94.

Welkie G and Miller G (1988). Riboflavin excretion from roots of iron-stressed and reciprocally grafted tobacco and tomato plants. J. Plant Nutr. 11: 691-700.

Wu H, Li L, Du J, Yuan Y, et al. (2005). Molecular and biochemical characterization of the Fe(III) chelate reductase gene family in Arabidopsis thaliana. Plant Cell Physiol. 46: 1505-1514.

Young ND, Debellé F, Oldroyd GE, Geurts R, et al. (2011). The Medicago genome provides insight into the evolution of rhizobial symbioses. Nature 480: 520-524. 